\title{
Pneumotorax e pneumopericárdio hipertensivo em cirurgia cardiotorácica*
}

\author{
RUI HADDAD', CARLOS EDUARDO TEIXEIRA LIMA², CARLOS HENRIQUE BOASQUEVISQUE ${ }^{3}$, \\ GUILHERME SARAIVA HADDAD ${ }^{4}$, TADEU DINIZ FERREIRA ${ }^{2}$
}

\begin{abstract}
RESUMO
São apresentados dois casos de pacientes com pneumotorax e pneumopericárdio hipertensivo, em pós-operatório de cirurgia cardiotorácica. Ambos tiveram abertura do pericárdio como um dos tempos cirúrgicos da operação inicial e apresentaram sintomas de tamponamento pericárdico como complicação. 0 tratamento foi uma drenagem pleural nos dois casos, que evoluíram para resolução do processo.
\end{abstract}

Descritores: Pneumopericário/etiologia; Pneumotórax/etiologia; Procedimentos cirúrgicos torácicos/efeitos adversos; Complicações pós-operatórias

\section{INTRODUÇÃO}

0 pneumopericárdio é definido como um acúmulo de ar ou gás no saco pericárdico. 0 pneumopericárdio hipertensivo é uma complicação rara, sendo que há poucos casos publicados na literatura. Ele é definido como um pneumopericárdio suficientemente grande para causar restrição diastólica e os sintomas característicos. As causas do pneumopericárdio incluem trauma torácico, ventilação mecânica (principalmente em crianças), propagação de doenças em órgãos contíguos, cirurgia cardio-torácica, infecção e pericardiocen- tese. ${ }^{(1)} 0$ pneumopericárdio hipertensivo é uma condição aguda e ameaçadora à vida que deve ser reconhecida de imediato e tratada para que se obtenha um desfecho favorável.

\section{Caso 1}

Um paciente do sexo masculino, com 40 anos, apresentando fibrilação atrial consultou um cardiologista. Ele não era tabagista e suas radiografias de tórax mostraram adenopatia hilar no lobo inferior direito. A tomografia computadorizada

\footnotetext{
* Trabalho realizado no departamento de Cirurgia Torácica Faculdade de Medicina da Universidade Federal do Rio de Janeiro - UFRJ - Rio de Janeiro (RJ) Brasil.

1. Doutorado em Cirurgia Torácica pela Faculdade de Medicina da Universidade Federal do Rio de Janeiro - UFRJ Rio de Janeiro (RJ) Brasil.

2.Mestre em Medicina pela Faculdade de Medicina da Universidade Federal do Rio de Janeiro - UFRJ - Rio de Janeiro (RJ) Brasil.

3. Chefe da Divisão de Cirurgia Torácica do IDT - Faculdade de Medicina da Universidade Federal do Rio de Janeiro UFRJ - Rio de Janeiro (RJ), Brasil.

4. Acadêmico do Curso de Medicina da Universidade Estácio de Sá -UNESA - Rio de Janeiro (RJ), Brasil.

Endereço para correspondência: Rui Haddad Av. Aquarela do Brasil 333 - Bloco 1 - Apto. 2401 - São Conrado - CEP:

22610-010 - Rio de Janeiro, RJ, Brasil

Recebido para publicação, em 27/2/05. Aprovado, após revisão, em 2/5/05.
} 
(TC) revelou uma massa central invadindo a veia pulmonar inferior e comprimindo o átrio esquerdo (Figura 1A). Uma lesão endobrônquica foi vista no brônquio lobar inferior direito e fez-se uma biopsia. Os resultados foram compativeis com carcinoma neuroendócrino de grandes células. 0 paciente foi submetido a três ciclos de quimioterapia à base de cisplatina e a radioterapia. As TCs subseqüentes (Figura 1B) e exames broncoscópicos revelaram uma resposta altamente positiva ao tratamento Ele foi submetido a uma pneumonectomia direita radical incluindo uma porção do contorno do átrio esquerdo (primeira margem de ressecção positiva na altura da veia pulmonar inferior) e do pericárdio para-hilar. 0 defeito pericárdico foi enxertado com pericárdio bovino e um orifício de $2 \mathrm{~cm}$ foi deixado na linha de sutura para permitir que fluidos ou ar pudessem sair livremente do saco pericárdico. 0 paciente recebeu alta no quinto dia de um pós-operatório curto e sem intercorrências. Dez dias depois, ele retornou ao hospital apresentando hipotensão postural e dor subesternal constante. A radiografia de tórax mostrou pneumopericárdio (Figure 1C), confirmado na TC, que também revelou múltiplas bolsas de ar e nível ar-líquido no espaço pleural, sugerindo fístula bronco-pleural (Figura 1D). Ele foi internado no hospital, um tubo torácico (36Fr) foi inserido no espaço pleural direito, e a broncoscopia mostrou uma pequenina $(1.0 \mathrm{~mm})$ fístula no coto brônquico. Não havia sinais ou sintomas de infecção e o líquido pleural estava claro, com menos do que 300 células, das quais $80 \%$ eram eosinófilos. 0 paciente não apresentou fuga aérea intra-operatória ou pós-operatória através do tubo torácico. Os antibióticos foram dados, e

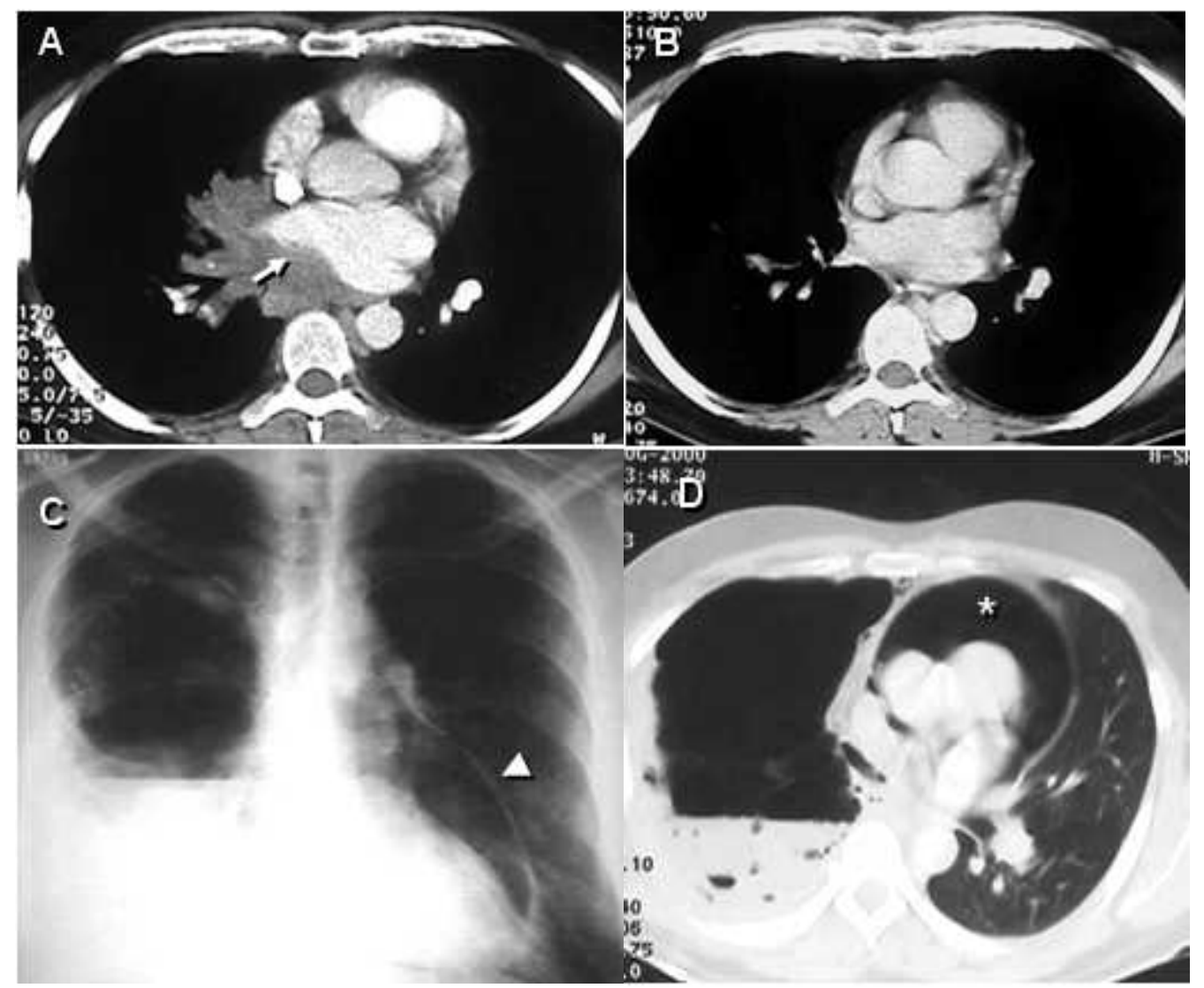

Figura 1 - A) Massa central invadindo a veia pulmonar inferior direita e comprimindo o átrio esquerdo (flecha). B) Resposta completa após três ciclos de quimioterapia; C) Radiografia de tórax com nível arlíquido no hemitórax direito e pneumopericárdio grande (ponta de flecha); D) Tomografia computadorizada com pneumopericárdio compressivo grande (*), nível ar-líquido no tórax direito e várias bolhas de ar no acúmulo. 


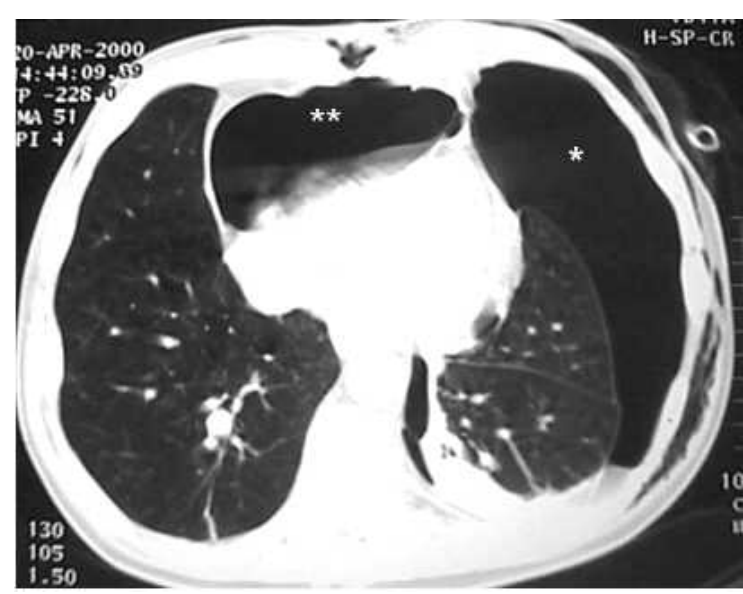

Figura 2 - Pneumotórax à esquerda (*) e pneumopericárdio hipertensivo $\left.{ }^{* *}\right)$

a evolução foi excelente. 0 tubo torácico foi retirado no décimo dia pós-operatório. 0 pneumopericárdio sintomático desapareceu imediatamente após uma drenagem pleural e não houve nenhum empiema ou outras complicações.

\section{Caso 2}

Um paciente do sexo masculino, com 64 anos, apresentando doença pulmonar obstrutiva crônica foi submetido a enxerto bypass de artéria coronária (EBAC) com anastomose de artéria mamária interna direita. Na unidade de terapia intensiva, duas horas após a extubação, ele apresentou pneumotórax espontâneo à esquerda, o qual foi controlado através da inserção de um cateter pigtail $14 \mathrm{Fr}$, após o quê o paciente melhorou. Porém, dois dias depois, ele apresentou desconforto respiratório e um segundo pneumotórax foi visto apesar do fato de que o cateter pigtail havia permanecido na cavidade pleural e encontrava-se desobstruído. Um segundo cateter pigtail foi inserido em posição anterior. 0 problema foi resolvido, e os cateteres foram removidos. No dia seguinte, o paciente apresentou hipotensão, taquicardia e dispnéia. Por motives técnicos, não foi possivel realizar um ecocardiograma. A TC mostrou um pneumotórax anterior esquerdo e um pneumopericárdio grande (Figura 2). Um tubo torácico 32 Fr foi inserido na cavidade pleural esquerda, e o problema foi totalmente resolvido. 0 paciente também foi submetido a uma pleurodese com talco em suspensão. A mesma solução simples (drenagem pleural) foi usada neste segundo caso.

\section{DISCUSSÃO}

0 pneumopericárdio hipertensivo na ausência de trauma torácico ou ventilação mecânica é uma entidade incomum. Ele geralmente envolve comunicação entre o pericárdio e a árvore traqueobroônquica ou o aparelho digestivo. Quando não há comunicação entre o saco pericárdico e a cavidade pleural, o tratamento de escolha é a drenagem pericárdica. Quando tal comunicação existe, a inserção de tubo torácico é preferível. Em pacientes submetidos a ressecção pulmonar intrapericárdica, o principal fator etiológico para pneumopericárdio hipertensivo é a fístula bronco-pleural, embora esta possa ser associada a pneumotórax ou a barotrauma decorrente de cirurgia cardíaca. As causas comuns de pneumopericárdio são trauma torácico penetrante, ventilação mecânica (barotrauma), propagação de doenças em órgãos contíguos, cirurgia cardio-torácica, procedimentos invasivos (pericardiocentese), infecção e comunicação anormal entre as vias aéreas e o pericárdio. (1) Nos dois casos aqui apresentados, os pacientes foram submetidos a procedimentos diferentes, mas ambos tiveram comunicação anormal entre as vias aéreas e o saco pericárdico associada a complicações pós-operatórias. Ambos os pacientes apresentaram pneumotórax, no primeiro caso, causada por uma pequena fístula bronco-pleural e, no segundo, causada por uma fístula alvéolopleural resultante de um barotrauma pós-EBAC. De fato, Capizzi et al. ${ }^{(2)}$ encontrou associação entre pneumotórax e pneumopericárdio hipertensivo em aproximadamente $87,5 \%$ dos casos avaliados. É importante ressaltar que o pneumopericárdio é mais prevalente em situações de não-trauma nas quais é necessário um suporte respiratório maior, tais como na síndrome do desconforto respiratório agudo ou no suporte respiratório neonatal. ${ }^{(3)}$ É também importante diferenciar o pneumopericárdio e o pneumomediastino, especialmente quando o pneumopericárdio é pequeno. Um indicador muito interessante, o sinal da cúpula diafragmática esquerda contínua, foi relatado em um caso de pneumopericárdio ${ }^{(4)}$ e pode ajudar no diagnóstico. Benedik et al. ${ }^{(5)}$ relatou um caso de tamponamento pneumopericárdico em pós-operatório de cirurgia bypass arterial coronariana. Os autores descobriram que a condição estava associada à ruptura de bolhas no saco pericárdico (as aderên- 
cias evitaram um pneumotórax concomitante) e a trataram usando toracotomia de emergência. Kim et al. ${ }^{(6)}$ publicou um caso de pneumopericárdio e pneumotórax causado pela ruptura de um tumor pulmonar cancerígeno no saco pericárdico. Brandenhoff et al. ${ }^{(7)}$ publicou dois casos de pneumopericárdio ocorridos após ressecção pulmonar, sendo que um deles era um caso de pneumopericárdio hipertensivo. Ambos os pacientes haviam estado sob suporte ventilatório prolongado e apresentaram fuga aérea anterior ao pneumopericárdio. Os autores trataram ambos os casos com toracotomia e abertura do pericárdio. Stuklis et al. ${ }^{(8)}$ publicou um caso de pneumopericárdio hipertensivo após lobectomia média que foi tratado usando a mesma técnica simples empregada em nossos dois casos, drenagem pleural.

\section{CONCLUSÃO}

A deterioração hemodinâmica aguda em pacientes com pneumotórax ou que necessitam de ventilação mecânica deve instigar uma investigação mais aprofundada e o tamponamento cardíaco deve ser ativamente excluído. Em casos de pneumopericárdio hipertensivo nos quais não há evidência de comunicação entre o saco pericárdi- co e a pleura, uma pericardiocentese de emergência ou drenagem deve ser feita. Contudo, se há tal evidência, uma drenagem pleural é indicada.

\section{REFERÊNCIAS}

1. Boyce SH, Corfield AR, McGuffie AC, Stevenson J, Rawlings D. Spontaneous tension pneumopericardium. Eur J Emerg Med. 2004; 11(3):181-4.

2. Capizzi PJ, Martin M, Bannon MP. Tension pneumopericardium following blunt injury. J Trauma. 1995; 39(4):775-80. Review.

3. Clouse WD, Dent DL, Stewart RM, Gray GA. Tension pneumopericardium from blunt chest trauma. Contemp Surg. 2003; 59(6):271-5.

4. Brander L, Ramsay D, Dreier D, Peter M, Graeni R. Continuous left hemidiaphragm sign revisited: a case of spontaneous pneumopericardium and literature review. Heart [serial on the Internet]. 2002; [cited 2005 Sep 17]: 88(4): [about 5p.]. Available from. http:// www.heartjnl.com/cgi/content/full/88/e5

5. Benedik J, Uchytil B, Cernosek J. Pneumopericardial tamponade after coronary artery bypass operation. Eur J Cardiothorac Surg. 2002;21(3):585-6. Review.

6. Kim Yl, Goo JM, Im JG. Concurrent pneumopericardium and pneumothorax complicating lung cancer: a case report. Korean J Radiol. 2000;1(2):118-20.

7. Brandenhoff P, Hoier-Madsen K, Struve-Christensen E. Pneumopericardium after pneumonectomy and lobectomy. Thorax. 1986:41(1):55-7.

8. Stuklis RG, Weeden DF. Images in cardiothoracic surgery. Pneumopericardium after lobectomy. Ann Thorac Surg. 2001;72(6):2144. 